\title{
Effects of Artificial Dawn and Morning Blue Light on Daytime Cognitive Performance, Well-being, Cortisol and Melatonin Levels
}

\author{
Virginie Gabel ${ }^{1}$, Micheline Maire ${ }^{1}$, Carolin F. Reichert ${ }^{1}$, Sarah L. Chellappa ${ }^{1}$, Christina Schmidt $^{1}$, \\ Vanja Hommes ${ }^{2}$, Antoine U. Viola ${ }^{1}$, and Christian Cajochen ${ }^{1}$. \\ ${ }^{1}$ Centre for Chronobiology, Psychiatric Hospital of the University of Basel, Basel, Switzerland and ${ }^{2} I T$ VitaLight I\&D PC \\ Drachten, Philips Consumer Lifestyle, Drachten, The Netherlands
}

\begin{abstract}
Light exposure elicits numerous effects on human physiology and behavior, such as better cognitive performance and mood. Here we investigated the role of morning light exposure as a countermeasure for impaired cognitive performance and mood under sleep restriction (SR). Seventeen participants took part of a $48 \mathrm{~h}$ laboratory protocol, during which three different light settings (separated by 2 wks) were administered each morning after two 6-h sleep restriction nights: a blue monochromatic LED (light-emitting diode) light condition (BL; 100 lux at $470 \mathrm{~nm}$ for $20 \mathrm{~min}$ ) starting $2 \mathrm{~h}$ after scheduled wake-up time, a dawn-simulating light (DsL) starting $30 \mathrm{~min}$ before and ending $20 \mathrm{~min}$ after scheduled wake-up time (polychromatic light gradually increasing from 0 to $250 \mathrm{lux}$ ), and a dim light (DL) condition for $2 \mathrm{~h}$ beginning upon scheduled wake time ( $<8$ lux). Cognitive tasks were performed every $2 \mathrm{~h}$ during scheduled wakefulness, and questionnaires were administered hourly to assess subjective sleepiness, mood, and wellbeing. Salivary melatonin and cortisol were collected throughout scheduled wakefulness in regular intervals, and the effects on melatonin were measured after only one light pulse. Following the first SR, analysis of the time course of cognitive performance during scheduled wakefulness indicated a decrease following $\mathrm{DL}$, whereas it remained stable following BL and significantly improved after DsL. Cognitive performance levels during the second day after SR were not significantly affected by the different light conditions. However, after both SR nights, mood and well-being were significantly enhanced after exposure to morning DsL compared with DL and BL. Melatonin onset occurred earlier after morning BL exposure, than after morning DsL and DL, whereas salivary cortisol levels were higher at wake-up time after DsL compared with BL and DL. Our data indicate that exposure to an artificial morning dawn simulation light improves subjective well-being, mood, and cognitive performance, as compared with DL and BL, with minimal impact on circadian phase. Thus, DsL may provide an effective strategy for enhancing cognitive performance, wellbeing, and mood under mild sleep restriction.
\end{abstract}

Keywords: Cognitive performance, cortisol, melatonin, morning light, sleep restriction, well-being

\section{INTRODUCTION}

Light exerts powerful non-image-forming (NIF) effects on behavioral and physiological functions, including hormonal secretion (Cajochen, 2005; Jung et al., 2010), sleep-wake regulation (for a review, see Chellappa et al., 2011a), cognitive function (Cajochen et al., 2011; Chellappa et al., 2011b), and its underlying cerebral correlates, encompassing cortical and subcortical brain regions (Vandewalle et al., 2009b). These light-dependent effects are to a large extent mediated by retinal photoreceptors containing the photopigment melanopsin, which are distinct from rods and cones (Hattar et al., 2002). Maximal sensitivity of the human alerting response and melatonin suppression to light occurs at the short-wavelength light (ca. $460-480 \mathrm{~nm}$ ), contrasting with the spectral sensitivity of classical visual photoreceptors (green light, $<550 \mathrm{~nm}$ ) (Cajochen et al., 2005; Lockley et al., 2006; Münch et al., 2006; Revell et al. 2006). Together with light's wavelength, other properties, such as intensity, duration, and timing, are crucial in determining its effects on human physiology and behavior (for a review, see Cajochen, 2007). Nighttime light exposure triggers melatonin suppression with concomitant reduction of subjective sleepiness and objective markers of sleepiness (e.g., waking electroencephalographic [EEG] theta activity, incidence of slow eye movements) (Cajochen et al., 2000; Chellappa et al,, 2011b, 2012; Lockley et al., 2006; Ruger et al., 2005). It has been suggested that these effects are mediated through melatonin's alerting effects and/or its resetting

Submitted December 17, 2012, Returned for revision March 27, 2013, Accepted March 28, 2013

*Antoine U. Viola and Christian Cajochen contributed equally to this work.

Correspondence: Antoine U. Viola, Centre for Chronobiology, Psychiatric Hospital of University of Basel, Wilhelm Klein-Strasse 27, CH-4018 Basel, Switzerland. Tel.: 00416132550 74; E-mail: antoine.viola@upkbs.ch 
properties on the endogenous circadian pacemaker (Chellappa et al., 2011a). Although the studies investigating the impact of daytime light are less abundant, three studies reported that bright white light exposure during daytime also enhances alertness (Phipps-Nelson et al., 2003; Ruger et al., 2005; Smolders et al., 2012). In the same vein, $20 \mathrm{~min}$ of daytime exposure to bright white light (ca. 7000 lux) increases task-related cortical activity while performing an oddball paradigm (Vandewalle et al., 2006). Bright white light sources are used as a therapeutic countermeasure against circadian rhythm sleep disorders (Zhu \& Zee, 2012) and depression, particularly seasonal depressive disorder (WirzJustice et al., 1986). Apart from its therapeutic use, bright light also improves well-being, vitality, and mood stability among healthy subjects working indoors in wintertime (Partonen \& Lonnqvist, 2000). Furthermore, chronic daytime exposure to blue-enriched moderateintensity light has the potential to improve visual comfort, alertness, sleepiness, and mood in the working environment (Viola et al., 2008).

More recently, the dynamics of light exposure have been shown to directly affect the impact of morning light exposure on sleep inertia, well-being, and cortisol levels (Gimenez et al., 2010; Van de Werken et al., 2010). The use of an artificial dawn light resulted in a significant reduction of sleep inertia complaints, which could not be explained by a shift in the dim light melatonin onset or timing of sleep offset (Gimenez et al., 2010). In another study, Van de Werken et al. (2010) showed that $30 \mathrm{~min}$ of an artificial dawn light (maximum 300 lux) prior to wake-up time was more efficient in alleviating sleep inertia and increasing subjective alertness compared to acute light exposure (300 lux) at wake-up time. However, they could not find significant effects on cognitive performance and did not confirm enhanced cortisol levels upon awakening after dawn, as observed earlier by Thorn et al. (2004). Although these initial studies clearly showed beneficial effects of an artificial dawn signal on sleep inertia and well-being, the design of the studies did not allow for testing how these acute effects of a dawn simulation light translate into daytime cognitive performance and well-being levels.

To address this question, we investigated the impact of morning artificial dawn simulation light exposure on alertness, well-being, mood, cognitive performance, and classical markers of the circadian timing system (melatonin and cortisol) and compared this effect with a dim light condition, as well as with a bright monochromatic blue light exposure of fixed intensity. The duration of light exposure during scheduled wakefulness (eyes open) was the same for each of the two experimental light conditions, but they differed in intensity, spectral composition, and the timing of administration. This latest aspect was motivated by the potential modality of using blue light not directly in the bedroom but at the beginning of the morning, possibly at work. One primary aim of this was to compare two morning light devices that are known for their effects on mood and cognitive performance, even though they do not share the same characteristics. The entire study protocol was performed under stringently controlled laboratory conditions, with two sleep restriction nights in order to test whether these light effects can counteract the detrimental effects of partial sleep loss on daytime alertness and cognitive performance, which is increasingly encountered in contemporary society. We hypothesized the following:

(1) As compared with the dim light condition, light exposure mimicking dawn will facilitate the wake-up process with respect to subjective perception of sleepiness, tension, and well-being and will have a beneficial impact on cognitive performance levels, especially in the time zone surrounding the wake-up period.

(2) As compared with the dim light condition, blue light exposure will increase subjective and objective neurobehavioral performance, which will be potentially sustained throughout the entire waking day.

(3) As maximal circadian phase-advancing properties have been attributed to short-wavelength light in the morning, particularly BL will lead to a advance in the assessed circadian phase markers (melatonin and cortisol).

\section{MATERIALS AND METHODS}

\section{Study Participants}

Study volunteers were recruited through advertisements at different local universities and internet sites in Switzerland, Germany, and France. The screening procedure began with a telephone interview, involving a detailed explanation of the study. All participants gave written informed consent. The study protocol, screening questionnaires, and consent forms were approved by the local ethics committee (EKBB/Ethikkommission beider Basel, Switzerland). They conformed to the Declaration of Helsinki and were performed in accordance with international ethical standards (Portaluppi et al., 2010). All applicants completed questionnaires about their sleep quality, life habits, and health state. These questionnaires comprised a consent form, a General Medical Questionnaire, the Beck Depression Inventory II (BDI-II) (Beck et al., 1961), the Epworth Sleepiness Scale (ESS) (Johns, 1991), the Horne-Östberg Morningness-Eveningness Questionnaire (MEQ) (Horne \& Östberg, 1976), the Munich Chronotype Questionnaire (MCTQ) (Roenneberg et al., 2003), and the Pittsburgh Sleep Quality Index (PSQI). Potential candidates with a PSQI score $>5$ were excluded from the study (Buysse et al., 1989). Further exclusion criteria were smoking, medication or drug consumption, body mass index $<19$ and $>28 \mathrm{~kg} / \mathrm{m}^{2}$, shiftwork within the last 3 months, transmeridian flights during the 3 months before the study, as well as medical and sleep disorders. Since our 
TABLE 1. Characteristics of the group of participants.

\begin{tabular}{lccc}
\hline Characteristic & Minimum & Maximum & Mean \pm SEM \\
\hline$n$ & & & 17 \\
Age & 20 & 33 & $23.12 \pm 0.82$ \\
Sleep time (h) & $21: 45$ & $2: 00$ & $23: 50 \pm 00: 19$ \\
Wake time (h) & $6: 00$ & $10: 00$ & $07: 57 \pm 00: 15$ \\
Sleep duration (h:min) & $7: 00$ & $9: 00$ & $08: 06 \pm 00: 08$ \\
BDI & 0 & 7 & $1.47 \pm 0.51$ \\
ESS & 1.5 & 14 & $5.79 \pm 1.01$ \\
MEQ & 29 & 74 & $54.35 \pm 2.64$ \\
MCTQ & 2.33 & 6.49 & $4.4 \pm 0.28$ \\
PSQI & 1 & 5 & $2.88 \pm 0.27$ \\
BMI & 20.75 & 25.76 & $22.86 \pm 0.35$ \\
\hline
\end{tabular}

$\mathrm{BDI}=$ Beck Depression Inventory; ESS = Epworth Sleepiness Scale; $\mathrm{MEQ}=$ Horne-Östberg Morningness-Eveningness Questionnaire; MCTQ $=$ Munich Chronotype Questionnaire; PSQI = Pittsburg

Sleep Quality Index; BMI = body mass index.

study protocol included two nights of partial sleep restriction (restriction to $6 \mathrm{~h}$ ), we also excluded participants with usual sleep durations of $<7 \mathrm{~h}$ and of $>9 \mathrm{~h}$ (Aeschbach et al., 1996), to minimize a possible confounding effect of sleep duration (Table 1).

One week before the study, participants were requested to refrain from alcohol, caffeine, and chocolate intake in order to level out the impact of these consumption behaviors on sleep and the other investigated variables reported here (e.g., subjective sleepiness). They were also instructed to keep a regular sleep-wake schedule (bedtimes and wake times within \pm 30 min of self-selected target time) for 1 wk prior to each study segment. Compliance was verified by wrist actigraphy (Actiwatch L; Cambridge Neurotechnologies, Cambridge, UK) and self-reported sleep logs. Eighteen young healthy men (20-33 years old; mean \pm SEM: $23.1 \pm 0.8)$ who fulfilled all inclusion criteria were selected to participate in the study. One participant could not be included in the analysis because of poor quality of the EEG recordings and noncompliance during cognitive testing. The remaining 17 volunteers had the following light treatment order: 3 DL-BL-DsL, 3 BL-DsL-DL, 3 DsL-DL-BL, 3 DL-DsL-BL, 3 BL-DL-DsL, and 2 DsL-BL-DL; where BL is a blue monochromatic LED (light-emitting diode) light condition (100lux at $470 \mathrm{~nm}$ for $20 \mathrm{~min}$ ) starting $2 \mathrm{~h}$ after scheduled wake-up time, DsL is a dawn-simulating light starting $30 \mathrm{~min}$ before and ending $20 \mathrm{~min}$ after scheduled wake-up time (polychromatic light gradually increasing from 0 to 250 lux), and DL is a dim light (DL) condition for $2 \mathrm{~h}$ beginning upon scheduled wake time $(<8$ lux). Six participants were morning (59 to 86), nine intermediate (42 to 58), and two evening (16 to 41) chronotypes, according to the MEQ, whereas for the MCTQ, there were seven morning ( 0 to 3.99), four intermediate (4 to 4.99), and six evening (5 to 9.5) chronotypes. A comprehensive urine toxicological analysis for drug abuse was carried out prior to the study, along with an ophthalmologic examination in order to exclude volunteers with visual impairments (visual field, color vision, pupillary reflex).

The study was carried out during the winter season (January to March) in Basel, Switzerland, to minimize the effects of outdoor ambient light levels. The laboratory setup comprised a balanced crossover design with three segments (one control condition and two experimental conditions), separated by an at least 2-wk intervening period. Participants remained in individual windowless and sound-attenuated bedrooms under controlled 40 lux light condition, except for the first $2 \mathrm{~h}$ after wake-up during the morning light exposure (see below). No information on time-of-day was given. Each study segment lasted $48 \mathrm{~h}$, comprising two sleep restriction (SR) nights (6h) adjusted to each participant's habitual bedtime followed by two 18-h scheduled waking days (Figure 1A). The light protocol for each session consisted of a 2-h dim light ( $<8$ lux) exposure after wake-up time followed by 40 lux until bedtime. The light treatment was administered after each sleeprestricted night, either with no additional light for the control condition, or with a blue light (BL; $2 \mathrm{~h}$ after wake-up, 20-min exposure of 100 lux of $470 \mathrm{~nm}$; full width at half maximum [FWHM] $27 \mathrm{~nm}$ ) with a photon density of $2.4 \mathrm{E} \pm 18$ photons $/ \mathrm{m}^{2} \cdot \mathrm{s}$ and a melanopic value of 4150 m-lux (goLITE BLU energy light; HF3330; Philips, Drachten, Netherlands) placed at $50 \mathrm{~cm}$ diagonally of the participant when he was sitting at the desk looking straight ahead (see Figure 1B for spectral composition), or with a DsL (polychromatic light gradually increasing from 0 to 250 lux during 30 min before wake-up time; the light remained around 250 lux for 20 min after wake-up time) placed near the bed at eye level (see Figure 1C for the time course of spectral composition). The illuminance, photon density, correlated color temperature and melanopic illuminance of the dawn simulation light, measured $45 \mathrm{~cm}$ from the device were as follows:

- $5 \mathrm{~min}$ after light onset: $1.2 \mathrm{lux}, 1.9 \mathrm{E} \pm 16 / \mathrm{m}^{2} \mathrm{~s}$, $1090 \mathrm{~K}, 0.2 \mathrm{~m}$-lux

- $15 \mathrm{~min}$ after light onset: $13 \mathrm{lux}, 1.4 \mathrm{E} \pm 17 / \mathrm{m}^{2} \mathrm{~s}$, $1500 \mathrm{~K}, 7.5 \mathrm{~m}$-lux

- $24 \mathrm{~min}$ after light onset: $78 \mathrm{lux}, 7.1 \mathrm{E} \pm 17 / \mathrm{m}^{2} \mathrm{~s}$, $2200 \mathrm{~K}, 120$ m-lux

- 30 min after light onset: 250 lux, $2.4 \mathrm{E} \pm 18 / \mathrm{m}^{2} \mathrm{~s}$, $2750 \mathrm{~K}, 620$ m-lux

\section{Subjective Assessment of Sleepiness, Well-being, and Mood}

Subjective sleepiness was assessed every hour, using the Karolinska Sleepiness Scale (KSS) (Akerstedt et al., 1994). Subjective tension was measured using a $100-\mathrm{mm}$ visual analog scale (VAS). Subjective well-being was assessed using a composite score calculated as follows: [VAS mood $+(100-$ VAS tension $)+(100-$ VAS physical comfort)]/3, according to Birchler-Pedross and colleagues (Birchler-Pedross et al., 2009). Subjective mood was investigated every $2 \mathrm{~h}$ on the Positive and Negative 
(A)

Time of Day (h)
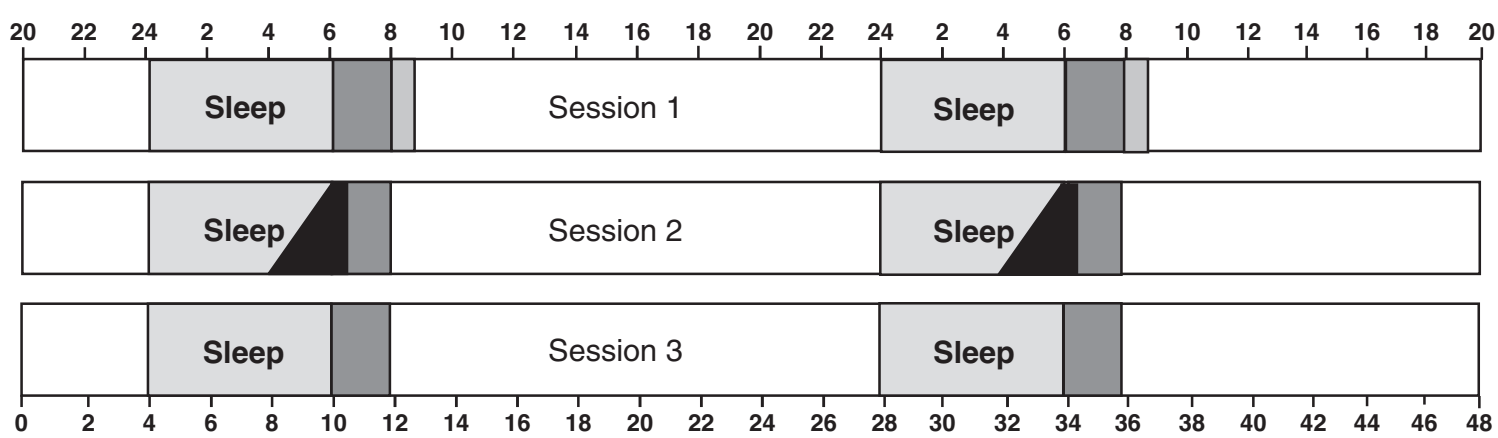

Elapsed Time into Protocol (h)

Continuous EEG, EOG, EMG, Heart Rate, Body Temperatures, Respiration etc.

Light Exposure: 8 lux

Light Exposure: 40 lux

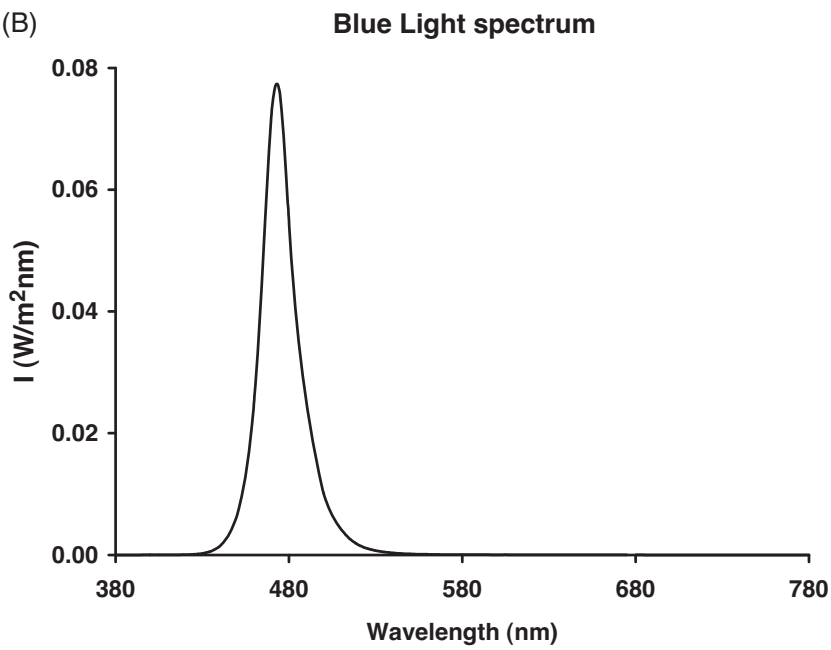

Blue monochromatic LEDs (goLite)
Dawn Simulating Light

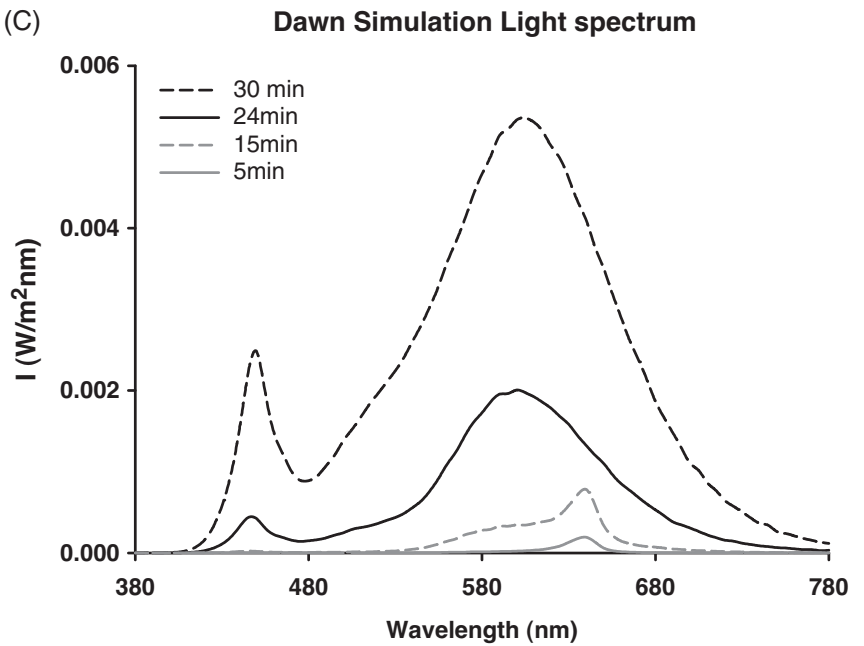

FIGURE 1. (A) Protocol design. Three conditions with different morning light exposures. Spectral composition (light wavelength by irradiance; $\mathrm{W} / \mathrm{m}^{2}$-nm) of the (A) blue light and the (B) dawn simulation light at 5 min (gray solid line), 15 min (gray dash line), 24 min (black solid line) and $30 \mathrm{~min}$ (black dash line).

Affect Scale (PANAS) (Watson et al., 1988). This questionnaire comprises two 10-item mood scales, and provides measures of positive affect (PA) and negative affect (NA) on a 5-point scale.

\section{Cognitive Performance}

Beginning $30 \mathrm{~min}$ after wake-up time, participants completed a 25 -min test battery every $2 \mathrm{~h}$. The battery included five cognitive tasks for sustained attention and executive functions: Sustained Attention to Response Task (SART; Robertson et al., 1997), Verbal 1-, 2-, and 3back (N-Back1-2-3; Kubat-Silman et al., 2002), and Paced Visual Serial Addition Task (PVSAT; Feinstein et al., 1994). To quantify the magnitude of the light effects on global cognitive performance, a composite score was computed by normalizing the data for each test separately (i.e., SART, PVSAT, N-Backl-2-3) and averaging it to yield a composite score for each volunteer for each 2-h period according to Viola and colleagues (Viola et al., 2007).

\section{Salivary Melatonin and Cortisol}

Saliva samples were scheduled during wakefulness every $30 \mathrm{~min}$ during the first $4 \mathrm{~h}$ after wake-up time and followed by hourly intervals until bedtime, starting the first evening upon arrival at the laboratory. Salivary samples were immediately frozen and kept at $-20^{\circ} \mathrm{C}$ until the melatonin and cortisol assays were conducted. A direct double-antibody radioimmunoassay was used for the melatonin assay (validated by gas chromatography-mass spectroscopy with an analytical least detectable dose of $0.65 \mathrm{pm} / \mathrm{mL}$; Bühlmann Laboratory, Schönenbuch, Switzerland; Weber et al., 1997). The minimum detectable dose of melatonin (analytical sensitivity) was determined to be $2 \mathrm{pg} / \mathrm{mL}$.

Cortisol was measured by ALPCO (ALPCO Diagnostics, Salem, NH, USA), using a direct salivary enzyme-linked immunosorbent assay (ELISA) for quantitative determination of cortisol. The sensitivity was 
TABLE 2. Analysis of variance for different variables for the time course of the study.

\begin{tabular}{llll}
\hline & \multicolumn{3}{c}{ Analysis of variance } \\
\cline { 2 - 4 } Variable & \multicolumn{1}{c}{ Light } & \multicolumn{1}{c}{ Time of day } & Light $\times$ Time of day \\
\hline Sleepiness & $F_{2,658}=4.70, p<0.01$ & $F_{14,658}=14.40, p<0.0001$ & $F_{28,658}=0.46, p=0.9927$ \\
Well-being & $F_{2,658}=12.00, p<0.0001$ & $F_{14,658}=6.20, p<0.0001$ & $F_{28,658}=0.47, p=0.9922$ \\
Tension & $F_{2,658}=13.20, p<0.0001$ & $F_{14,658}=3.40, p<0.0001$ & $F_{28,658}=0.53, p=0.9786$ \\
Positive mood & $F_{2,702}=3.17, p=0.0426$ & $F_{14,702}=8.33, p<0.0001$ & $F_{28,702}=0.96, p=0.5291$ \\
Negative mood & $F_{2,702}=8.21, p=0.0003$ & $F_{14,702}=0.44, p=0.9617$ & $F_{28,702}=0.42, p=0.9965$ \\
Cognitive performance & $F_{2,658}=10.31, p<0.0001$ & $F_{14,658}=0.32, p=0.9920$ & $F_{28,658}=0.40, p=0.9978$ \\
\hline
\end{tabular}

$1.0 \mathrm{ng} / \mathrm{mL}$ and intra-assay coefficient of variances amounts to $10.3 \%$ for baseline values $6.6 \mathrm{ng} / \mathrm{mL}$.

\section{Statistical Analysis}

For all analysis, the statistical package SAS (version 9.1; SAS Institute, Cary, NC, USA) was used. Statistical analyses were carried out for each variable (subjective sleepiness, subjective well-being, positive and negative affects, and cognitive performance) separately with the mixed-model analysis of variance for repeated measures (PROC MIXED), with within factors "light condition" (dim light [DL] versus blue light [BL] versus dawn simulation light [DsL]) and "time-of-day" (all assessed time points). The rationale for this approach was that time-of-day was deemed as a continuum, and thus a better form to illustrate the temporal dynamics of all our dependent variables. The time course of the melatonin profiles were analyzed for each day (SR1 and SR2) separately, since melatonin onsets were only available for the baseline evening and in the evening after SR1. Cortisol analysis was also done separately for SR1 and SR2, since we intended to analyze the wake-up response after both sleep restriction nights. Contrasts were assessed with the LSMEANS statement, and degrees of freedom were corrected with the Kenward-Rogers contrasts. The Wilcoxon-Mann-Whitney test was used for post hoc comparisons, since not all data reached the criterion for a normal distribution. Alpha adjustment for multiple comparisons was applied according to CurranEverett (Curran-Everett, 2000).

For the analyses of the dim light melatonin onset (DLMO) following exposure to either DL, BL, or DsL, data were $z$-scored to provide a normalized comparison of amplitudes across each individual (Zeitzer et al., 1999). The individual $z$-score adjustment provides a mean at 0 with a variation of 1 . For each melatonin profile, we derived a DLMO using a fixed threshold of 0 . The DLMO was defined as the time when melatonin levels hit and exceeded the threshold. The time the melatonin levels crossed the threshold was determined by linear interpolation between the points immediately below and above the threshold on the day following exposure to either BL or DsL relative to melatonin onset on the day prior to light exposure (baseline levels of melatonin under dim light conditions) (Zeitzer et al., 1999).

\section{RESULTS}

\section{Subjective Assessment of Sleepiness, Well-being, and Mood}

With respect to subjective sleepiness, we observed a significant main effect of "light condition" and "timeof-day" (Table 2). Analysis revealed no significant influence of light exposition on subjective sleepiness after the first sleep restriction (SR). However, after the second SR, participants felt subjectively sleepier following BL exposure compared with the DsL and DL conditions (Figure 2, first panel). Post hoc analysis revealed a significant difference between the DsL and the DL exposure at $10 \mathrm{~h}$ of elapsed time awake (ca. 16:00 $\mathrm{h}$ the afternoon) after the second night of sleep restriction and between the $\mathrm{BL}$ and the DL exposure at $4 \mathrm{~h}$ of elapsed time awake (ca. $10 \mathrm{~h}$ the morning) after the second night of sleep restriction (Wilcoxon nonparametric test).

Concerning well-being, we observed a significant main effect for the factors "light condition" and "timeof-day" (Table 2). Furthermore, considering the light effect, analyses revealed that the participants felt generally better during the entire study after a DsL exposure compared to a BL or a DL exposure. Compared with the BL exposure, well-being after the DsL improved during approximately $7 \mathrm{~h}$ after the first SR night. After BL exposure, well-being levels were maintained stable compared with DL condition. After the second SR night, the level of well-being was likewise increased after a DsL exposure throughout all the day. Post hoc analysis revealed a significant difference between the DsL and the DL exposure at 10 and $12 \mathrm{~h}$ of elapsed time awake (ca. 16:00 and 18:00 h the afternoon) after the second night of sleep restriction (Wilcoxon non-parametric test) (Figure 2, second panel).

A similar profile was observed with respect to subjective tension (main effect of "light condition" and "time-of-day"; Table 2). Subjective tension was lower after a DsL exposure compared with BL or DL exposure across all the experiment. Post hoc analysis revealed a significant difference between the DsL and the DL exposure at 6,10 , and $12 \mathrm{~h}$ of elapsed time awake (ca. 12:00, 16:00 and 18:00 h the afternoon) after the second night of sleep restriction (Wilcoxon nonparametric test) (Figure 2, third panel).

Concerning the mood scales, we observed significant effects of "light condition" and "time-of-day" when 


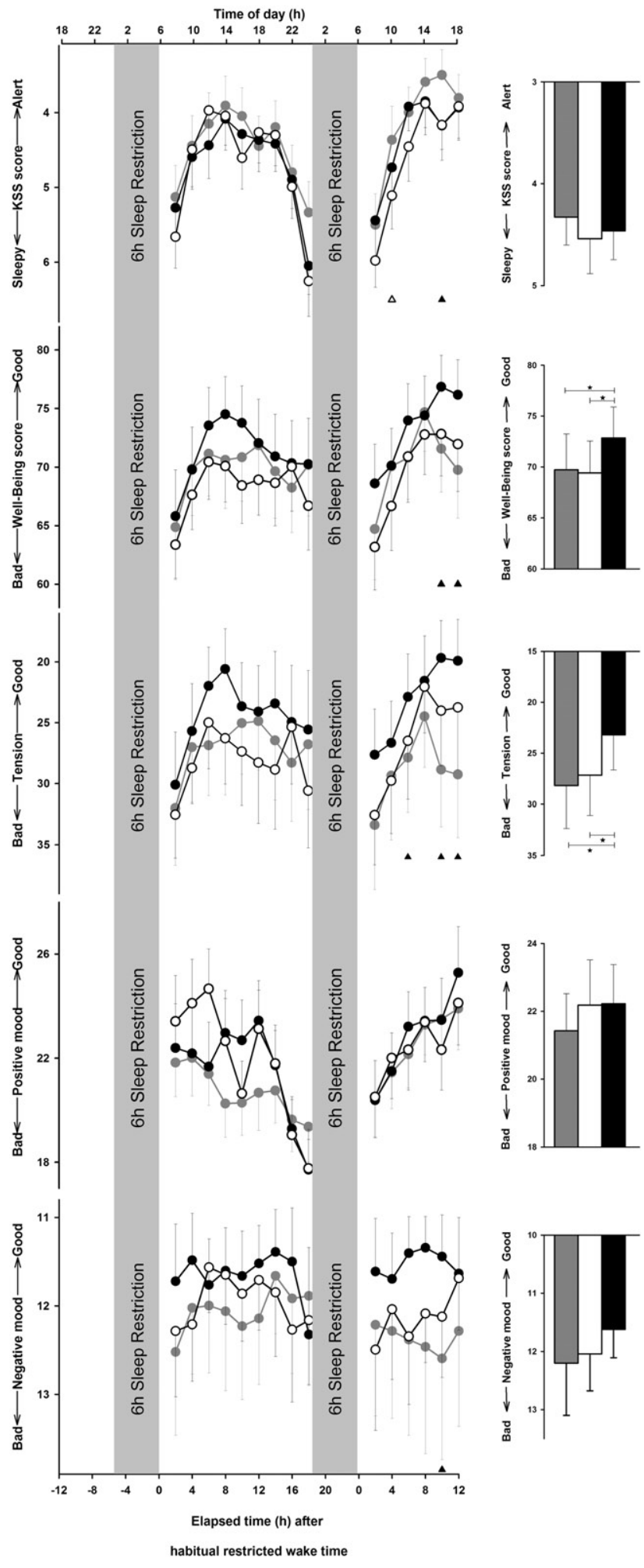

FIGURE 2. Time course (left panel) and mean of all the time points (right panel) of (top to bottom) subjective sleepiness, subjective well-being, tension, positive mood, and negative mood in 17 participants under dim light (dark gray lines), monochromatic blue LEDs (black lines with white circles), or dawn simulation light (black lines with black circles). Data are plotted as a mean for each 2-h bin relative to elapsed time (h) after wake time, and the error bars represent the standard error of the mean. Post hoc comparisons were carried out using the Wilcoxon-Mann-Whitney test between the DsL and the DL $(\boldsymbol{\Delta}, p<0.05)$ and between the BL and the DL $(\Delta, p<0.05)$. looking at the overall time course. DsL and BL exposures helped maintaining mood after the first SR night, whereas under the dim light condition, positive mood was significantly lower. After the second SR night, positive mood was equal across all light settings (Figure 2, fourth panel). However, the post hoc analysis did not reveal significant light effects.

For the negative mood scale, only a main effect of "light condition" was found. A reverse pattern was also observed: although the light condition did not affect negative mood after the first sleep restriction night, DsL was able to decrease negative mood over the day following the second sleep restriction night. Post hoc analysis revealed a significant difference between the DsL and the DL exposure at $10 \mathrm{~h}$ of elapsed time awake (ca. 16:00 $\mathrm{h}$ the afternoon) after the second night of sleep restriction (Wilcoxon non-parametric test) (Figure 2, fifth panel).

\section{Cognitive Performance}

Analysis of the composite score of cognitive performance revealed a significant main effect of "light condition" when looking at the overall time course. Nevertheless, analysis did not reveal a general significant effect concerning the light treatment. Following the first sleep restriction night, cognitive performance was significantly better following morning DsL exposure compared with morning BL exposure, in which performance did not differ compared to DL. After the second SR night, these differences were no longer significant between DsL and DL. Interestingly, cognitive performance started on a higher level the day following the second SR compared to the level before this SR night under the latter light conditions, whereas levels remained similar for the blue light condition until the end of the protocol. Post hoc analysis revealed a significant difference between the DsL and the DL exposure at $16 \mathrm{~h}$ of elapsed time awake (ca. 22:00 h the afternoon) after the first night of sleep restriction (Wilcoxon non-parametric test) (Figure 3).

\section{Salivary Melatonin}

During the baseline evening, before the first SR and light intervention, melatonin levels were equal across all conditions. However, analysis of the melatonin profile during the first evening after morning light exposure yielded a significant main effect of "light condition" $\left(F_{2,280}=9.3, p=0.0001\right)$ and "time-of-day" $\left(F_{6,280}=62.7\right.$, $p<0.0001)$, as well as an interaction "light condition" versus "time-of-day" $\left(F_{10,280}=2.0, p<0.04\right)$. Although melatonin levels were not affected by the DsL, neither after the first, nor after the second SR; morning BL exposure elicited a phase advance in salivary melatonin. Melatonin secretion increased earlier after a BL exposure than after a DsL or DL exposure (Figure 4A). Congruently, we found a main effect of "light condition" $\left(F_{2,27.4}=5.4, p=0.01\right)$ for the DLMO, which was significantly earlier after morning BL $(21: 20 \mathrm{~h} \pm 19 \mathrm{~min})$ 


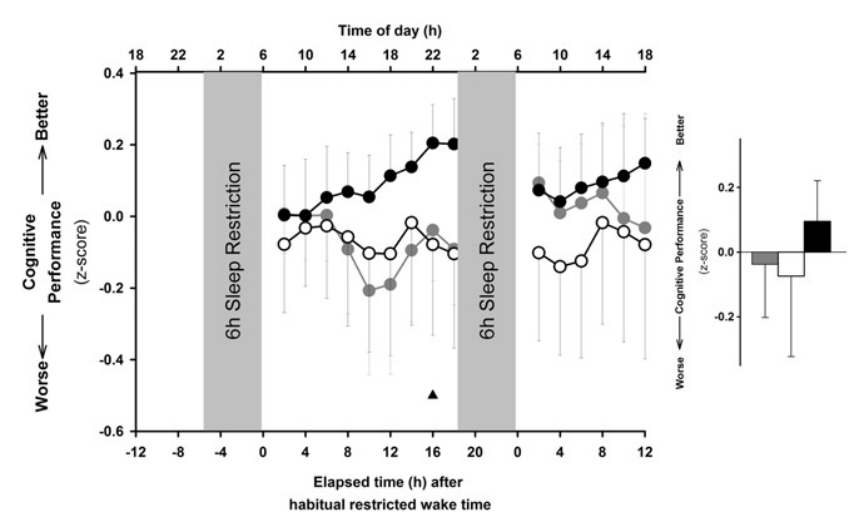

FIGURE 3. Time course (left panel) and mean of all the time points (right panel) of the composite of cognitive performance in 17 participants under dim light (dark gray lines), monochromatic blue LEDs (black lines with white circles), or dawn simulation light (black lines with black circles). Data are plotted as a mean for each 2-h bin relative to elapsed time (h) after wake time, and the error bars represent the standard error of the mean. Post hoc comparisons were carried out using the Wilcoxon-Mann-Whitney test between the DsL and the DL $(\boldsymbol{\Lambda}, p<0.05)$ and between the BL and the DL $(\Delta, p<0.05)$.

exposure compared to DL (21:50 $\mathrm{h} \pm 23 \mathrm{~min} ; p=0.003)$ and DsL (21:38 $\mathrm{h} \pm 17 \mathrm{~min} ; p=0.05)$ exposure.

\section{Salivary Cortisol}

Comparable to melatonin profile, cortisol levels were similar across sessions on the first evening, before intervening SR and light application. After light exposure and SR, a main effect of "light condition" was observed, irrespective of whether the morning after the first $\left(F_{2,363}=3.3, \quad p=0.0383\right) \quad$ or second $\left(F_{2,381}=3.5\right.$, $p=0.0317)$ SR night was considered. Compared to the DL condition, cortisol levels decreased during the first evening after 13 to $16 \mathrm{~h}$ of elapsed time awake (i.e., corresponding time-of-day: $18: 00 \mathrm{~h}$ to $22: 00 \mathrm{~h}$ ) after a morning BL exposure but not after a DsL exposure $\left(F_{2,270}=8.9, p=0.0002\right)$. Additionally, during the first hour since awakening, a significant increase in cortisol levels upon wake-up with the DsL was observed during both mornings after SR (mean \pm SEM: $\mathrm{DL}=20.35 \pm 1.43 \mathrm{ng} / \mathrm{mL} ; \quad \mathrm{BL}=22.96 \pm 1.49 \mathrm{ng} / \mathrm{mL} ;$ $\mathrm{DsL}=31.99 \pm 2.13 \mathrm{ng} / \mathrm{mL})$ compared to the two other conditions $\left(F_{2,32}=15.65, p<0.0001\right)$ (Figure $\left.4 \mathrm{~B}\right)$.

\section{DISCUSSION}

Here we investigated whether the exposure to artificial light sources, differing in their dynamics and spectral sensitivity, can facilitate the waking-up process and allow the improvement of subjective parameters of wellbeing, sleepiness, and tension, as well as cognitive performance throughout a sustained period of wakefulness, following sleep restriction. Our data suggest that morning exposure to dawn simulation light can significantly improve subjective perception of well-being, mood, and tension, as well as cognitive performance.

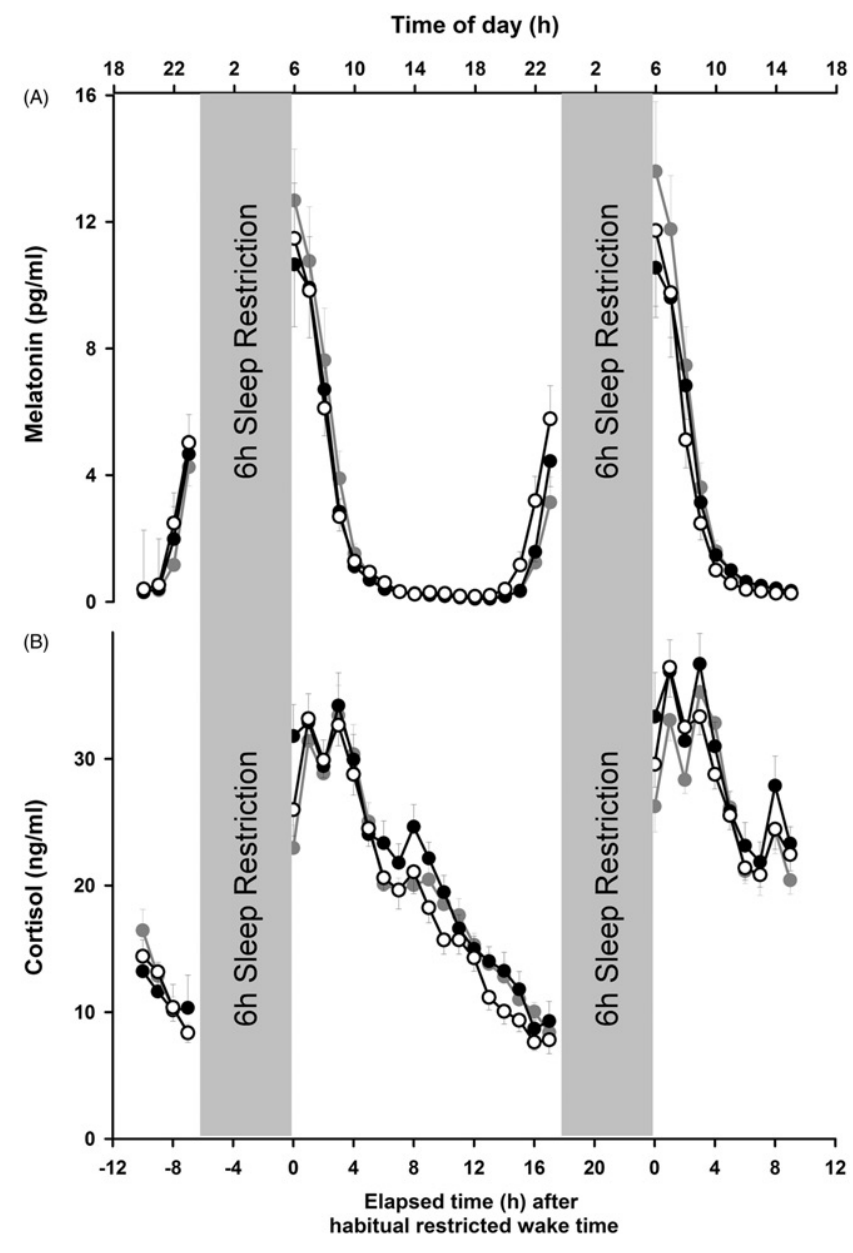

FIGURE 4. Time course of salivary (A) melatonin and (B) cortisol profiles in 17 participants under dim light (dark gray lines), monochromatic blue LEDs (black lines with white circles), or dawn simulation light (black lines with black circles). Data are plotted as a mean for each 2-h bin relative to elapsed time (h) after wake time, and the error bars represent the standard error of the mean.

Importantly, this effect can persist up to $18 \mathrm{~h}$ after light exposure. In parallel, dawn light simulation did not change the circadian phase of cortisol and melatonin profiles, in contrast to exposure to blue monochromatic light-emitting diodes (LEDs). In other words, although the light effects on classical markers of circadian phase showed maximal sensitivity to short-wavelength light exposure (blue monochromatic LEDs), the sustained effects on subjective well-being, mood, tension, and cognitive performance were mostly driven by the exposure to a broadband polychromatic light source simulating dawn.

The strengths of this study are the strictly controlled laboratory conditions and the selection criteria of the volunteers (sleep-wake times were similar for all participants), which allowed us to carefully quantify and analyze the effects of dawn simulation light and blue monochromatic light in contrast to a dim light condition, and to thus conclude that our light-induced effects were not driven exclusively by prior differences in circadian phase and sleep pressure levels. 
Our data are in line with previous observations indicating that $30 \mathrm{~min}$ of artificial dawn light exposure in the morning hours improves subjective well-being during the time window of sleep inertia (Gimenez et al., 2010). Similarly, we found that light exposure around wake-up time counteracted the detrimental effects of sleep inertia, resulting in higher subjective well-being, but also in more positive (respectively less negative) mood as assessed by a validated affect scale (PANAS). Importantly, our data show for the first time that these effects are sustained over a whole waking day after one sleep-restricted night (for mood measures) and are even still operational after two nights of partial sleep restriction for subjective well-being and tension.

Beneficial effects of light on mood may be mediated through long-term circadian effects (Avery et al., 2001; Lewy et al., 1987). Another possible neuroanatomical modulator for these effects is the amygdala, a key component of the limbic system, which encompasses brain areas related to emotion processing, with direct projections from melanopsin-containing intrinsic photosensitive retinal ganglion cells (ipRGCs), and is acutely affected by light exposure (Vandewalle et al., 2010).

Our data suggest that dawn simulation light could induce facilitation of the wake-up process and allows a sustained beneficial effect throughout the entire waking day. Interestingly, the observed increase of subjective well-being, tension, and mood was associated with higher cortisol levels after wake-up with the dawn simulation light, in comparison with dim light and monochromatic blue LED exposure, which was most likely applied too late to induce any cortisol response. Light after wake-up (Scheer \& Buijs, 1999), and especially dawn simulation light (Thorn et al., 2004), has previously been reported to affect cortisol levels upon awakening. Furthermore, bright light seems to trigger maximal effects on cortisol secretion when applied during the upward portion of cortisol secretion, i.e., in the early morning hours, close to wake-up time (Jung et al., 2010). It may be assumed that these increased cortisol levels upon wake-up reflect a light-induced stress response. However, we observed that our volunteers felt subjectively more relaxed after DsL exposure as compared to BL and DL. Alternatively, and in line with what we observed at the subjective level, DsL before and after wake-up could facilitate the wake-up process per se and positively affect the process of sleep inertia by increased cortisol levels immediately after wake-up. Such an effect might be mediated by the suprachiasmatic nuclei (SCN), which have direct multisynaptic neural pathways to the adrenal cortex (Buijs et al., 1999).

Dawn simulation light increased cognitive performance levels over the first day after SR. This beneficial effect was no longer maintained after the second SR night. It may thus be assumed that artificial dawn simulation light is beneficial for the maintenance of cognitive performance, but only if homeostatic sleep pressure, as challenged by SR, is not sufficiently high to be "unresponsive" to light. Recent functional magnetic resonance imaging (fMRI) studies propose a cerebral network underlying beneficial non-image-forming effects of light on cognition (Vandewalle et al., 2009a). It is suggested that the light irradiance signal impinges onto the SCN, indirectly communicating with the brainstem locus coeruleus, which in turn presents thalamic and cortical connections allowing modulation over widespread cortical networks implicated in successful cognitive performance (Vandewalle et al., 2009b). However, little is known about the magnitude, dynamics, and regional brain distribution of such nonimage-forming effects and how properties, such as dose, duration, intensity, and for instance different light dynamics impact on the above mentioned networks (Chellappa et al., 2011a). Subcortical regions are thought to be more promptly activated and display short-lasting responses to light, whereas the long-lasting and widespread cortical task-related brain responses appear when light exposure is longer and at a higher intensity (Perrin et al., 2004). It may be speculated that the duration of light exposure, here indexed by $30 \mathrm{~min}$ of gradually increasing polychromatic light exposure (dawn simulation light) followed by 20 min of moderate light exposure (light duration of $50 \mathrm{~min}$ ), results in such long-lasting optimal cognitive performance across the day.

Spectral sensitivity of non-image-forming effects of light on circadian physiology has repeatedly been shown to be highest in the short-wavelength range of the visible light spectrum (Brainard et al., 2001; Gooley et al., 2010). In our study, we observed that a 20-min exposure to monochromatic blue LEDs $2 \mathrm{~h}$ after wake-up did not affect subjective sleepiness, tension, and cognitive performance compared to a dim light condition. However, we did not have a control light condition at equal time-of-day with the same duration as comparison. We also did not measure during the blue light exposure but only $40 \mathrm{~min}$ later for the KSS and $10 \mathrm{~min}$ later for mood and cognition. Moreover, most of the studies reporting specific spectral sensitivity of blue light on alertness in humans administered light either during nighttime or the evening hours (Cajochen, 2007; Chellappa et al., 2011a), except for the fMRI study (Vandewalle et al., 2006).

Concomitant to the absence of effects at the subjective and cognitive levels after BL exposure as compared with the DL control condition, we observed a significant phase advance in the circadian phase markers of melatonin and cortisol after morning blue LED exposure. Our results are in line with previously observed phase advances for melatonin after $6.5 \mathrm{~h}$ bright light exposure centered after the core body temperature nadir (Khalsa et al., 2003). Exposure to intermittent blue LED light (three 30 -min pulses over $2 \mathrm{~h}$ ) over 3 days induced melatonin phase shifts, such that the phase advances extend later in the day and the phase delays start earlier 
relative to white light (Revell et al., 2012). Very recently, a phase response curve (PRC) for short-wavelength (monochromatic blue $480 \mathrm{~nm}$ ) light was constructed to assess DLMO phase shifts (Ruger et al., 2013). Exposure to $6.5 \mathrm{~h}$ of $480 \mathrm{~nm}$ light indicated fitted maximum delays and advances of -2.6 and $1.3 \mathrm{~h}$, respectively. Furthermore, the $6.5 \mathrm{~h}$ of $480 \mathrm{~nm}, 11.2$ lux light PRC resulted in approximately $75 \%$ of the response of the $6.7 \mathrm{~h}$ of 10000 lux white light PRC. This capacity for blue light (during the phase-advancing portion of the human PRC) to significantly advance DLMO may thus explain our earlier melatonin onset following a 20 -min morning light exposure to monochromatic blue LED relative to DSL and DL. One likely explanation is the higher irradiance and melanopic values in the short wavelength, since the mechanisms involved in melatonin suppression are mostly sensitive to this wavelength (Brainard et al., 2001, 2008). Blue light impinges onto the non-image-forming pathway, involving melanopsincontaining intrinsic photosensitive retinal ganglion cells (ipRGCs), which then modulates responses in the ventrolateral preoptic nucleus (VLPO) and the SCN via a specialized non-image-forming retinohypothalamic tract with direct neuronal connections to the SCN (Tsai et al., 2009). Given that the SCN is directly involved in the circadian timing system, with direct neuronal projections to the pineal gland (central site for melatonin production), nocturnal melatonin secretion would then phase advance, as would be predicted by the classical phase response curve of light to the circadian system (Khalsa et al., 2003; Minors et al., 1991).

Nevertheless, the magnitude of which our data can be extrapolated to real-life settings should be viewed with caution, since under those conditions, other concomitant effects such as social constraint, exposure to outdoor light, and different types of artificial lighting, may mask, minimize, or perhaps even enhance the effects of our current results. Future studies under reallife settings may be required to probe the extent to which morning light devices may impact on mood and cognitive performance, particularly on a long-term perspective.

\section{CONCLUSION}

Our data indicate that exposure to artificial morning dawn simulation light improves subjective perception of well-being and mood, as well as cognitive performance, under conditions of mild sleep restriction. Concomitantly, it did not result in phase advances of cortisol and melatonin profiles, as induced by the blue light, thus resulting in a minimal impact on the circadian timing system. In a broader context, these light conditions may provide an effective rationale for enhancing performance and mood in individuals who experience conditions of mild sleep restriction.

\section{ACKNOWLEDGEMENTS}

We thank Dr. Götz for medical screenings; Claudia Renz, Marie-France Dattler, and Giovanni Balestrieri for their help in data acquisition; and Amandine Valomon for her help in recruiting volunteers and the volunteers to participating.

\section{DECLARATION OF INTEREST}

This research was supported by Philips Consumer Lifestyle, Drachten, The Netherlands.

The authors report no conflicts of interest. The authors alone are responsible for the content and writing of the paper.

\section{REFERENCES}

Aeschbach D, Cajochen C, Landolt H, Borbely AA. (1996). Homeostatic sleep regulation in habitual short sleepers and long sleepers. Am J Physiol, 270, R41-53.

Akerstedt T, Hume K, Minors D, Waterhouse J. (1994). The subjective meaning of good sleep, an intraindividual approach using the Karolinska Sleep Diary. Percept Mot Skills, 79, 287-96.

Avery DH, Eder DN, Bolte MA, et al. (2001). Dawn simulation and bright light in the treatment of SAD: A controlled study. Biol Psychiatry, 50, 205-16.

Beck AT, Ward CH, Mendelson M, et al. (1961). An inventory for measuring depression. Arch Gen Psychiatry, 4, 561-71.

Birchler-Pedross A, Schroder CM, Munch M, et al. (2009). Subjective well-being is modulated by circadian phase, sleep pressure, age, and gender. J Biol Rhythms, 24, 232-42.

Brainard GC, Hanifin JP, Greeson JM, et al. (2001). Action spectrum for melatonin regulation in humans: Evidence for a novel circadian photoreceptor. J Neurosci, 21, 6405-12.

Brainard GC, Sliney D, Hanifin JP, et al. (2008). Sensitivity of the human circadian system to short-wavelength (420-nm) light. J Biol Rhythms, 23, 379-86.

Buijs RM, Wortel J, Van Heerikhuize JJ, et al. (1999). Anatomical and functional demonstration of a multisynaptic suprachiasmatic nucleus adrenal (cortex) pathway. Eur J Neurosci, 11, $1535-44$.

Buysse DJ, Reynolds CF, Monk TH, et al. (1989). The Pittsburgh Sleep Quality Index: A new instrument for psychiatric practice and research. Psychiatry Res, 28, 193-213.

Cajochen C. (2005). Sleep disruption in shift work and jet lag: The role of the circadian timing system. Praxis (Bern 1994), 94, 1479-83.

Cajochen C. (2007). Alerting effects of light. Sleep Med Rev, 11, 453-64.

Cajochen C, Brunner DP, Krauchi K, et al. (2000). EEG and subjective sleepiness during extended wakefulness in seasonal affective disorder: Circadian and homeostatic influences. Biol Psychiatry, 47, 610-17.

Cajochen C, Frey S, Anders D, et al. (2011). Evening exposure to a light-emitting diodes (LED)-backlit computer screen affects circadian physiology and cognitive performance. J Appl Physiol, $110,1432-8$.

Cajochen C, Munch M, Kobialka S, et al. (2005). High sensitivity of human melatonin, alertness, thermoregulation, and heart rate to short wavelength light. J Clin Endocrinol Metab, 90, 1311-16.

Chellappa SL, Gordijn MC, Cajochen C. (2011a). Can light make us bright? Effects of light on cognition and sleep. Prog Brain Res, 190, 119-33. 
Chellappa SL, Steiner R, Blattner P, et al. (2011b). Non-visual effects of light on melatonin, alertness and cognitive performance: Can blue-enriched light keep us alert? PLoS ONE, 6, e16429.

Chellappa SL, Viola AU, Schmidt C, et al. (2012). Human melatonin and alerting response to blue-enriched light depend on a polymorphism in the clock gene PER3. J Clin Endocrinol Metab, 97, E433-7.

Curran-Everett D. (2000). Multiple comparisons: Philosophies and illustrations. Am J Physiol Regul Integr Comp Physiol, 279, R1-8.

Feinstein A, Brown R, Ron M. (1994). Effects of practice of serial tests of attention in healthy subjects. J Clin Exp Neuropsychol, $16,436-47$.

Gimenez MC, Hessels M, Van de Werken M, et al. (2010). Effects of artificial dawn on subjective ratings of sleep inertia and dim light melatonin onset. Chronobiol Int, 27, 1219-41.

Gooley JJ, Rajaratnam SM, Brainard GC, et al. (2010). Spectral responses of the human circadian system depend on the irradiance and duration of exposure to light. Sci Transl Med, 2, 31 ra33.

Hattar S, Liao HW, Takao M, et al. (2002). Melanopsin-containing retinal ganglion cells: Architecture, projections, and intrinsic photosensitivity. Science, 295, 1065-70.

Horne JA, Östberg O. (1976). A self-assessment questionnaire to determine morningness-eveningness in human circadian rhythms. Int J Chronobiol, 4, 97-110.

Johns MW. (1991). A new method for measuring daytime sleepiness: The Epworth sleepiness scale. Sleep, 14, 540-5.

Jung CM, Khalsa SB, Scheer FA, et al. (2010). Acute effects of bright light exposure on cortisol levels. J Biol Rhythms, 25, 208-16.

Khalsa SB, Jewett ME, Cajochen C, Czeisler CA. (2003). A phase response curve to single bright light pulses in human subjects. J Physiol, 549, 945-52.

Kubat-Silman AK, Dagenbach D, Absher JR. (2002). Patterns of impaired verbal, spatial, and object working memory after thalamic lesions. Brain Cogn, 50, 178-93.

Lewy AJ, Sack RL, Miller LS, Hoban TM. (1987). Antidepressant and circadian phase-shifting effects of light. Science, 235, $352-4$.

Lockley SW, Evans EE, Scheer FA, et al. (2006). Short-wavelength sensitivity for the direct effects of light on alertness, vigilance, and the waking electroencephalogram in humans. Sleep, 29, $161-8$.

Minors DS, Waterhouse JM, Wirz-Justice A. (1991). A human phase-response curve to light. Neurosci Lett, 133, 36-40.

Münch M, Kobialka S, Steiner R, et al. (2006). Wavelengthdependent effects of evening light exposure on sleep architecture and sleep EEG power density in men. Am J Physiol Regul Integr Comp Physiol, 290, R1421-8.

Partonen T, Lonnqvist J. (2000). Bright light improves vitality and alleviates distress in healthy people. J Affect Disord, 57, $55-61$.

Perrin F, Peigneux P, Fuchs S, et al. (2004). Nonvisual responses to light exposure in the human brain during the circadian night. Curr Biol, 14, 1842-6.

Phipps-Nelson J, Redman JR, Dijk DJ, Rajaratnam SM. (2003). Daytime exposure to bright light, as compared to dim light, decreases sleepiness and improves psychomotor vigilance performance. Sleep, 26, 695-700.

Portaluppi F, Smolensky MH, Touitou Y. (2010). Ethics and methods for biological rhythm research on animals and human beings. Chronobiol Int, 27, 1911-29.

Revell VL, Arendt J, Fogg LF, Skene DJ. (2006). Alerting effects of light are sensitive to very short wavelengths. Neurosci Lett, 399, 96-100.
Revell VL, Molina TA, Eastman CI. (2012). Human phase response curve to intermittent blue light using a commercially available device. J Physiol, 590, 4859-68.

Robertson IH, Manly T, Andrade J, et al. (1997). 'Oops!': Performance correlates of everyday attentional failures in traumatic brain injured and normal subjects. Neuropsychologia, 35, 747-58.

Roenneberg T, Wirz-Justice A, Merrow M. (2003). Life between clocks: Daily temporal patterns of human chronotypes. J Biol Rhythms, 18, 80-90.

Ruger M, Gordijn MC, Beersma DG, et al. (2005). Weak relationships between suppression of melatonin and suppression of sleepiness/fatigue in response to light exposure. J Sleep Res, 14, 221-7.

Ruger M, St Hilaire MA, Brainard GC, et al. (2013). Human phase response curve to a single $6.5 \mathrm{~h}$ pulse of short-wavelength light. J Physiol, 591, 353-63.

Scheer FA, Buijs RM. (1999). Light affects morning salivary cortisol in humans. J Clin Endocrinol Metab, 84, 3395-8.

Smolders KC, de Kort YA, Cluitmans PJ. (2012). A higher illuminance induces alertness even during office hours: Findings on subjective measures, task performance and heart rate measures. Physiol Behav, 107, 7-16.

Thorn L, Hucklebridge F, Esgate A, et al. (2004). The effect of dawn simulation on the cortisol response to awakening in healthy participants. Psychoneuroendocrinology, 29, 925-30.

Tsai JW, Hannibal J, Hagiwara G, et al. (2009). Melanopsin as a sleep modulator: Circadian gating of the direct effects of light on sleep and altered sleep homeostasis in Opn4(-I-) mice. PLoS Biol, 7, e1000125.

Van de Werken M, Gimenez MC, De Vries B, et al. (2010). Effects of artificial dawn on sleep inertia, skin temperature, and the awakening cortisol response. J Sleep Res, 19, 425-35.

Vandewalle G, Balteau E, Phillips C, et al. (2006). Daytime light exposure dynamically enhances brain responses. Curr Biol, 16, 1616-21.

Vandewalle G, Archer SN, Wuillaume C, et al. (2009a). Functional magnetic resonance imaging-assessed brain responses during an executive task depend on interaction of sleep homeostasis, circadian phase, and PER3 genotype. J Neurosci, 29, 7948-56.

Vandewalle G, Maquet P, Dijk DJ. (2009b). Light as a modulator of cognitive brain function. Trends Cogn Sci, 13, 429-38.

Vandewalle G, Schwartz S, Grandjean D, et al. (2010). Spectral quality of light modulates emotional brain responses in humans. Proc Natl Acad Sci USA, 107, 19549-54.

Viola AU, Archer SN, James LM, et al. (2007). PER3 polymorphism predicts sleep structure and waking performance. Curr Biol, 17, 613-18.

Viola AU, James LM, Schlangen LJ, Dijk DJ. (2008). Blue-enriched white light in the workplace improves self-reported alertness, performance and sleep quality. Scand J Work Environ Health, 34, 297-306.

Watson D, Clark LA, Tellegen A. (1988). Development and validation of brief measures of positive and negative affect: The PANAS scales. J Pers Soc Psychol, 54, 1063-70.

Weber JM, Schwander JC, Unger I, Meier D. (1997). A direct ultrasensitive RIA for the determination of melatonin in human saliva: Comparison with serum levels. J Sleep Res, 26, 757.

Wirz-Justice A, Bucheli C, Schmid AC, Graw P. (1986). A dose relationship in bright white light treatment of seasonal depression. Am J Psychiatry, 143, 932-3.

Zeitzer JM, Daniels JE, Duffy JF, et al. (1999). Do plasma melatonin concentrations decline with age? Am. J Med, 107, 432-6.

Zhu L, Zee PC. (2012). Circadian rhythm sleep disorders. Neurol Clin, 30, 1167-91. 